\title{
Aferição do pH de diferentes agentes clareadores indicados para o clareamento intracoronário
}

\author{
- João Felipe Besegato Departamento de Odontologia Restauradora, Universidade Estadual de Londrina, \\ Londrina, PR, Brasil - Gabriela dos Santos Ribeiro Rocha Departamento de Odontologia Restauradora, Universidade \\ Estadual de Londrina, Londrina, PR, Brasil - Marlene de Sousa Amorim Departamento de Odontologia Restauradora, \\ Universidade Estadual de Londrina, Londrina, PR, Brasil • Fabio Martins Salomão Departamento de Odontologia \\ Restauradora, Faculdade de Odontologia de Araçatuba, Araçatuba, SP, Brasil • Daniel Poletto Departamento de \\ Odontologia, Universidade Paranaense, Francisco Beltrão, PR, Brasil • Kelly Limi Aida Departamento de Odontologia \\ Restauradora, Faculdade de Odontologia de Araçatuba, Araçatuba, SP, Brasil • Pablo Guilherme Caldarelli Departamento \\ de Medicinal Oral e Odontologia Infantil, Universidade Estadual de Londrina, Londrina, PR, Brasil • Márcio Grama \\ Hoeppner Departamento de Odontologia Restauradora, Universidade Estadual de Londrina, Londrina, PR, Brasil
}

RESUMO | Objetivo: Aferir os valores de pH de diferentes produtos clareadores indicados para uso na técnica intracoronária em diferentes intervalos de tempo. Materiais e métodos: Para cada grupo experimental (G), foram preparadas cinco amostras $(\mathrm{n}=5)$ : G1 - água destilada (AD); G2 - peróxido de hidrogênio (H2O2) a 30\%; G3 - perborato de sódio (PbS) + $\mathrm{AD}$; G4 $\mathrm{PbS}+\mathrm{H}_{2} \mathrm{O} 2$ a 30\%; G5 - percarbonato de sódio $(\mathrm{PcS})+\mathrm{AD}$; e $\mathrm{G} 6-\mathrm{PcS}+\mathrm{H}_{2} \mathrm{O} 2$ a 30\%. O pH foi determinado por um pHmetro digital, em diferentes intervalos de tempo: imediatamente após a manipulação (To), 24 horas (T1) e 168 horas após a manipulação (T2). Os dados obtidos foram submetidos à análise estatística por meio do teste de Kruskal-Wallis, seguido pelo teste de Mann Whitney para comparações múltiplas entre os grupos. Para verificar o efeito do tempo em cada grupo, foi aplicado o teste de Friedman. Resultados: Na avaliação do efeito do tempo em cada grupo, observou-se que G2 apresentou comportamento ácido, enquanto os demais grupos exibiram valores de pH próximos da neutralidade ou alcalinos. Conclusão: $\mathrm{H}_{2} \mathrm{O} 230 \%$ foi o único agente que apresentou comportamento ácido em todos os tempos de avaliação, e a mistura $\mathrm{PcS}+\mathrm{H} 2 \mathrm{O}$ foi a que apresentou os maiores valores de $\mathrm{pH}$.

DESCRITORES | Clareamento Dental; Clareadores Dentários; Concentração de Íons de Hidrogênio.

ABSTRACT | pH measure of bleaching agents indicated to intracoronal bleaching - Objective: to measure pH values of bleaching agents that are indicated to intracoronal bleaching technique in different time intervals. Methods: Each group (G) received five samples $(\mathrm{n}=5)$ : G1 - distilled water (AD); G2 - hydrogen peroxide (H2O2) 30\%; $\mathrm{G}_{3}$ - sodium perborate (PbS) + AD; $\mathrm{G}_{4}-\mathrm{PbS}+\mathrm{H}_{2} \mathrm{O}_{2} 30 \%$; $\mathrm{G}_{5}-$ sodium percarbonate $(\mathrm{PcS})+\mathrm{AD}$; and $\mathrm{G} 6-\mathrm{PcS}+\mathrm{H}_{2} \mathrm{O} 230 \%$. pH values were stated using a digital pHmeter, in different time intervals: immediately after handling (To), 24 hours (T1) and 168 hours after handling (T2). The results were submitted to statistical analysis through Kruskal-Wallis and Mann Whitney tests, in this order, allowing multiple comparisons among the groups. To verify the effect of time in each group, Friedman test was applied. Results: In the evaluation of the effect of time in each group, it was observed that G2 presented acid behavior, while the other groups exhibited values close to neutrality or alkaline. Conclusions: $\mathrm{H}_{2} \mathrm{O} 230 \%$ was the only agent that showed acidic behavior in every evaluation time. Meanwhile, $\mathrm{PcS}+\mathrm{H}_{2} \mathrm{O}$ had the highest $\mathrm{pH}$ values.

DESCRIPTORS | Tooth Bleaching; Tooth Bleaching Agents; Hydrogen-Ion Concentration.

AUTOR CORRESPONDENTE | • João Felipe Besegato Curso de Odontologia, Universidade Estadual de Londrina • Rua Pernambuco, 540, Centro, Londrina, PR, Brasil • 86010-120 E-mail: besegato@outlook.com

- Received Feb. 7, 2018 • Accepted Feb. 12, 2018

- Dol http://dx.doi.org/10.11606/issn.2357-8041.clrd.2018.143185 


\section{INTRODUÇÃO}

O clareamento dentário intracoronário é indicado para dentes tratados endodonticamente e que apresentam alterações de cor, as quais podem ser causadas por fatores intrínsecos ou extrínsecos, como tratamento endodôntico insatisfatório, medicação intracanal e traumatismo dentário ${ }^{1-3}$. Quando corretamente indicado, o procedimento apresenta-se como uma opção conservadora, que promove o clareamento satisfatório da estrutura dentária em um curto período e a baixo custo ${ }^{4,5}$.

$\mathrm{O}$ peróxido de hidrogênio $\left(\mathrm{H}_{2} \mathrm{O}_{2}\right)$, em concentrações variadas, é o agente clareador mais utilizado em diferentes técnicas, isolado e/ ou em associação ao perborato de sódio $(\mathrm{PbS})^{6,7}$. Para catalisar sua decomposição, considerada lenta em condições normais, foi indicado o uso em associação a uma fonte geradora de luz/ calor $^{8,9}$.

O perborato de sódio $(\mathrm{PbS})$ é um agente oxidante em pó, que, na presença de um ácido ou veículo aquoso, decompõe-se em metaborato de sódio, $\mathrm{H}_{2} \mathrm{O}_{2}$ e oxigênio ${ }^{6}$. Embora seja uma opção consagrada no clareamento intracoronário, o $\mathrm{PbS}$ pode ser substituído pelo percarbonato de sódio (PcS), agente oxidante originado da reação entre $\mathrm{H}_{2} \mathrm{O}_{2}$ e carbonato de sódio, muito empregado na indústria química e têxtil ${ }^{10,11}$. No entanto, poucos são os estudos que referenciam ou avaliam o PcS como um agente clareador dentário intracoronário ${ }^{12}$.

A concentração dos agentes clareadores é o fator mais comumente estudado; entretanto, poucos trabalhos investigam o valor do $\mathrm{pH}$, sua influência no processo de clareamento dentário e seus efeitos sobre a estrutura dentária ${ }^{13}$. Assim, o objetivo deste experimento foi avaliar e comparar, em diferentes intervalos de tempo, o potencial hidrogeniônico $(\mathrm{pH})$ de diferentes agentes clareadores indicados para o clareamento intracoronário.

\section{MATERIAIS E MÉTODOS}

Foi avaliado o pH de duas substâncias: água destilada (AD) - solução controle - e $\mathrm{H}_{2} \mathrm{O}_{2}$ a $30 \%$, isoladamente e em associação ao $\mathrm{PbS}$ ou PcS $\left(2 \mathrm{Na}_{2} \mathrm{CO}_{3} \cdot 3 \mathrm{H}_{2} \mathrm{O}_{2}\right.$, manipulado no laboratório de pesquisa da empresa Biodinâmica Química e Farmacêutica Ltda., Ibiporã, Paraná, Brasil, a qual também foi responsável pelo fornecimento das substâncias). As medições foram feitas em diferentes intervalos de tempo (To - inicial, T1 - 24 horas, e T2 - 168 horas) e, para cada grupo experimental (G), foram preparadas cinco amostras (N), como indica o Quadro 1.

Quadro 1 | Grupos experimentais.

\begin{tabular}{|c|c|c|c|c|}
\hline Grupo & $\mathrm{N}$ & Materiais & Fator em estudo & Tempo \\
\hline $\begin{array}{c}\text { G1 } \\
\text { (Controle) }\end{array}$ & 5 & Água destilada (25 ml/frasco) & \multirow{6}{*}{$\mathrm{pH}$} & \multirow{6}{*}{$\begin{array}{c}\text { T0 - inicial } \\
\text { T1 - } 24 \text { horas } \\
\text { T2 - } 168 \text { horas }\end{array}$} \\
\hline G2 & 5 & Peróxido de hidrogênio a 30\% (25 ml/frasco) & & \\
\hline G3 & 5 & Perborato de sódio (70 g) + água destilada (35 ml) & & \\
\hline G4 & 5 & Perborato de sódio (70 g) + peróxido de hidrogênio a 30\% (35ml) & & \\
\hline G5 & 5 & Percarbonato de sódio (70 g) + água destilada (35 ml) & & \\
\hline G6 & 5 & Percarbonato de sódio (70 g) + peróxido de hidrogênio a 30\% (35 ml) & & \\
\hline
\end{tabular}


Os materiais avaliados foram acondicionados em frascos plásticos de armazenamento de filme fotográfico, com tampa e de cor escura, para evitar exposição à luz durante todas as etapas do experimento. Eles foram previamente lavados com detergente neutro e água corrente, secos e identificados de acordo com o grupo correspondente e, no grupo, por ordem de leitura do $\mathrm{pH}$, de 1 a 5 .

Para a pesagem do $\mathrm{PbS}$ e $\mathrm{PcS}$, foi utilizada uma balança semianalítica da marca Gehaka, modelo BG 2000, com precisão de um centésimo de grama. Para aferição do $\mathrm{pH}$, em todos os intervalos de tempo, utilizou-se um pHmetro digital (Gehaka, Modelo PG 1800), cuja calibração foi realizada com duas soluções eletrolíticas da marca Digimed (São Paulo, SP), com pH 6,86 (DM-51A) e 4,o (DM-51B). A cada intervalo de tempo, a aferição foi feita imergindo o bulbo do aparelho nas substâncias e pastas clareadoras. Depois, o bulbo foi lavado com água deionizada à temperatura ambiente. Entre as aferições, o bulbo do pHmetro digital era mantido submerso em solução de cloreto de potássio, para evitar sua descalibragem.

Para análise dos valores de $\mathrm{pH}$ dos diferentes materiais, em função do tempo, os dados foram previamente submetidos ao teste de normalidade e homocedasticidade, e utilizou-se o teste de Friedman para verificar o efeito do tempo em cada grupo. Para comparações múltiplas entre os grupos, empregou-se o teste de Kruskal-Wallis, seguido pelo teste de Mann-Whitney. Adotou-se nível de significância de $5 \%$, e todos os dados foram analisados pelo programa estatístico SPSS 15.0 (SPSS, Chicago, IL, USA).

\section{RESULTADOS}

A Tabela 1 informa os valores das medianas de $\mathrm{pH}$ aferidos nos diferentes grupos, em função do tempo. Na avaliação do efeito do tempo em cada grupo, observaram-se comportamentos opostos dos grupos G2 e G3 em comparação aos
G4, G5 e G6, visto que os primeiros apresentaram diminuição significativa do $\mathrm{pH}$, enquanto os demais exibiram aumento significativo.

Tabela 1 | Aferição do pH (mediana - range) de substâncias clareadoras em função do tempo.

\begin{tabular}{c|c|c|c}
\multirow{2}{*}{ Grupos } & \multicolumn{3}{|c}{ Tempos } \\
\cline { 2 - 4 } & T0 & T1 & T2 \\
G1 & 7,60 & 6,47 & 6,64 \\
\hline Controle) & $(0,23)^{\mathrm{Aa}}$ & $(0,31)^{\mathrm{Ba}}$ & $(1,65)^{\mathrm{Ca}}$ \\
\hline \multirow{2}{*}{ G2 } & 6,13 & 5,93 & $5,81(0,21)^{\mathrm{Ca}}$ \\
\hline \multirow{2}{*}{ G3 } & $(0,07)^{\mathrm{Ab}}$ & $(0,05)^{\mathrm{Bb}}$ & 10,92 \\
& 11,31 & 11,17 & $(0,06)^{\mathrm{Cb}}$ \\
\hline \multirow{2}{*}{ G4 } & $(0,14)^{\mathrm{Ac}}$ & $(0,17)^{\mathrm{Bc}}$ & 9,76 \\
& 7,09 & 8,09 & $(0,03)^{\mathrm{Cc}}$ \\
\hline \multirow{2}{*}{ G5 } & $(0,04)^{\mathrm{Ad}}$ & $(0,13)^{\mathrm{Bd}}$ & 13,28 \\
& 12,64 & 12,81 & $(0,09)^{\mathrm{Cd}}$ \\
\hline \multirow{2}{*}{ G6 } & $(0,06)^{\mathrm{Ae}}$ & $(0,03)^{\mathrm{Be}}$ & 13,08 \\
& 10,90 & 11,64 & $(0,06)^{\mathrm{Ce}}$ \\
\hline
\end{tabular}

* Letras maiúsculas diferentes, diferença significativa entre os tempos em cada grupo (Teste de Friedman, $p<0,05$ )

*Letras minúsculas diferentes, diferença significativa entre os grupos em cada tempo (Kruskal-Wallis e Mann-Whitney, $p<0,05$ )

No grupo controle, observou-se diminuição significativa do $\mathrm{pH}$ após 24h, mas houve aumento no período de $168 \mathrm{~h}$. Após a análise dos grupos nos três períodos, verificou-se que G2 apresentou pH significativamente inferior quando comparado aos demais; em $\mathrm{G}_{5}$, verificaram-se valores de $\mathrm{pH}$ superiores aos outros em todos os intervalos de tempo.

\section{DISCUSSÃO}

Os resultados evidenciaram que todas as soluções apresentaram $\mathrm{pH}$ próximo da neutralidade $(\mathrm{pH} 7)$ ou valores alcalinos, com exceção de G2, devido ao caráter ácido do $\mathrm{H}_{2} \mathrm{O}_{2}{ }^{14}$. $\mathrm{O}$ valor do $\mathrm{pH}$ dos agentes clareadores é um fator relevante, pois um meio tornado ácido a partir de uma reação de oxidação pode provocar alterações químicas e/ou morfológicas na estrutura dentária, se utilizado na técnica de clareamento intracoronária. Muitas dessas alterações podem ser prejudiciais, como a reabsorção cervical externa de dentes despolpados ${ }^{13,15,16}$. 
$\mathrm{O}$ comportamento ácido do $\mathrm{H}_{2} \mathrm{O}_{2} 30 \%$ pode desmineralizar o tecido dentinário e aumentar sua permeabilidade, expor as fibras colágenas e diminuir a microdureza do substrato ${ }^{17,18}$. Com o aumento da permeabilidade dentinária, a difusão do $\mathrm{H}_{2} \mathrm{O}_{2}$ e, consequentemente, o seu contato com os tecidos periodontais de suporte, podem ser desencadeadas reações inflamatórias, aumento da atividade osteoclástica e reabsorção cervical externa ${ }^{10,19}$.

O PbS é um agente oxidante em pó, estável em ambiente seco, que pode ser utilizado em associação a $\mathrm{H}_{2} \mathrm{O}$ ou $\mathrm{H}_{2} \mathrm{O}_{2}$, e a pasta resultante da mistura $\mathrm{PbS}+\mathrm{H}_{2} \mathrm{O}_{2}$ é comumente utilizada como curativo de demora na técnica de clareamento em dentes despolpados ${ }^{4}$. Neste estudo, G3 e G4 apresentaram maiores valores de $\mathrm{pH}$ quando comparados a G1 e G2, respectivamente. O PbS, em contato com um veículo líquido, decompõe-se em peróxido de hidrogênio e metaborato de sódio, o qual é responsável pelo aumento do $\mathrm{pH}$ da mistura durante a degradação e ação de clareamento ${ }^{14}$.

Em relação à indicação e comportamento dos produtos avaliados, as misturas $\mathrm{PbS}+\mathrm{H}_{2} \mathrm{O}$ e $\mathrm{PbS}$ $+\mathrm{H}_{2} \mathrm{O}_{2}$ são efetivas no clareamento dentário ${ }^{20,21}$. A mistura $\mathrm{PbS}+\mathrm{H}_{2} \mathrm{O}_{2}$ produz maior quantidade de radicais hidroxilas e, consequentemente, requer menor número de sessões clínicas para a obtenção do resultado desejado. Por sua vez, embora necessite de maior tempo clínico, $\mathrm{PbS}+\mathrm{H}_{2} \mathrm{O}$ é um protocolo mais seguro para o clareamento de dentes despolpados ${ }^{22}$.

Todas as misturas testadas apresentaram valores médios de $\mathrm{pH}$ alcalino, e a mistura $\mathrm{PcS}+\mathrm{H}_{2} \mathrm{O}$ exibiu alcalinidade mais significativa. O PcS, quando dissolvido em água, resulta na produção de carbonato de sódio e $\mathrm{H}_{2} \mathrm{O}_{2}$, além de proporcionar um meio alcalino e oxidativo. Assim, devido a sua capacidade oxidativa, o PcS também é um agente indicado para o clareamento intracoronário. As misturas de $\mathrm{PcS}+$ $\mathrm{H}_{2} \mathrm{O}$ e $\mathrm{PcS}+\mathrm{H}_{2} \mathrm{O}_{2}$ foram mais efetivas que a mistura $\mathrm{PbS}+\mathrm{H}_{2} \mathrm{O}$ no clareamento de dentes despolpados ${ }^{10}$; no entanto, mais estudos são necessários para avaliar o uso do PcS como agente clareador intracoronário e, em especial, a longevidade dos dentes clareados e os resultados imediatos obtidos ${ }^{6,12}$.

\section{CONCLUSÃO}

A partir dos resultados, podemos concluir que $\mathrm{H}_{2} \mathrm{O}_{2} 30 \%$ foi o único agente que apresentou comportamento ácido em todos os tempos de avaliação, enquanto a mistura $\mathrm{PcS}+\mathrm{H}_{2} \mathrm{O}$ apresentou os maiores valores de $\mathrm{pH}$. Do ponto de vista clínico, a utilização de agentes alcalinos na técnica intracoronária contribuiu para minimizar os danos causados no tecido dentinário.

\section{REFERÊNCIAS}

1. Kim JH, Kim Y, Shin SJ, Park JW, Jung IY. Tooth Discoloration of immature permanent incisor associated with triple antibiotic therapy: a case report. J Endod. 2010 Jun;36(6):1086-91. doi: 10.1016/j.joen.2010.03.03.

2. Krastl G, Allgayer N, Lenherr P, Filippi A, Taneja P, Weiger R. Tooth discoloration induced by endodontic materials: a literature review. Dent Traumatol. 2013 Feb;29(1):2-7. doi: 10.1111/j.1600-9657.2012.01141.x.

3. Patil AG, Hiremath V, Kumar RS, Sheetal A, Nagaral S. Bleaching of a non-vital anterior tooth to removetheintrinsic discoloration. J Nat Sci Biol Med. 2014 Jul-Dec;5(2):476-9. doi: 10.4103/09769668.136269.

4. Attin T, PaqueF,Ajam F, Lennon AM. Review of thecurrentstatus of tooth whitening with the walking bleach technique. Int Endod J. 2003 May;36(5):313-29.

5. TanoE, OtsukiM, KatoJ,SadrA,IkedaM,TagamiJ.Effects of 405 nm diodelaser on titanium oxidebleaching activation. Photomed LaserSurg. 2012Nov;30(11):648-54. doi:10.1089/pho.2012.3273.

6. Tano E, Otsuki M, Kato J, Sadr A, Ikeda M, Tagami J. Effects of $405 \mathrm{~nm}$ diode laser on titanium oxide bleaching activation. Photomed Laser Surg. 2012 Nov;30(11):648-54. doi: 10.1089/pho.2012.3273.

7. Plotino G, Buono Plotino G, Buono L, Grande NM, Pameijer $\mathrm{CH}$, Somma F. Nonvital tooth bleaching: a review of the literature and clinical procedures. J Endod. 2008 Apr;34(4):394-407. doi: 10.1016/j.joen.2007.12.020. 
8. Basson RA, Grobler SR, Kotze TJ, Osman Y. Guidelines for the selection of tooth whitening products amongst those available on the market. SADJ. 2013 Apr;68(3):1229.

9. Gurgan S, Cakir FY, Yazici E. Different light-activated inoffice bleaching systems: a clinical evaluation. Lasers Med Sci. 2010 Nov;25(6):817-22. doi: 10.1007/s10103-009-0688-x.

10. Gonçalves RS, Costa CA, Soares DG, Santos PH, Cintra LT, Briso AL. Effect of different light sources and enamel preconditioning on color change, $\mathrm{H}_{2} \mathrm{O}_{2}$ penetration, and cytotoxicity in bleached teeth. Oper Dent. 2016 Jan-Feb;41(1):83-92. doi: 10.2341/14-364-L.

11. Kaneko J, Inoue S, Kawakami S, Sano H. Bleaching effect of sodium percarbonate on discolored pulpless teeth in vitro. J Endod. 2000 Jan;26(1):25-8. doi: 10.1097/00004770200001000-00006.

12. Kuga MC, ReisJMSN, FabricioS, Bonetti-Filho I, deCamposEA, Faria G. Fracture strength of incisor crowns after intracoronal bleaching with sodium percarbonate. Dent Traumatol. 2012 Jun;28(3):238-42. doi: 10.1111/j.1600-9657.2011.01077.x.

13. Zimmerli B, Jeger F, Lussi A. Bleaching of nonvital teeth: a clinically relevant literature review. Schweiz Monatsschr Zahnmed. 2010;120(4):306-20.

14. Sun L, Liang S, Sa Y, Wang Z, Ma X, Jiang T, et al. Surface alteration of human tooth enamel subjected to acidic and neutral 30\% hydrogen peroxide. J Dent. 2011 Oct;39(10):686-92. doi: 10.1016/j.jdent.2011.07.011.

15. Barcellos DC, Borges AB, Silva RC, Ribeiro LM, Pucci CR, Torres CRG. pH-changes during intracoronal bleaching: an in vivo study. J Contemp Dent Pract. 2011 Mar 1;12(2):109-13.
16. Sulieman M, Addy M, Macdonald E, Rees JS. A safety study in vitro for the effects of an in-office bleaching system on the integrity of enamel and dentine.J Dent. 2004 Sep;32(7):581-90. doi: 10.1016/j.jdent.2004.06.003.

17. Xu B, Li Q, Wang Y. Effects of $\mathrm{pH}$ values of hydrogen peroxide bleaching agents on enamel surface properties. Oper Dent. 2011 Sep-Oct;36(5):554-62. doi: 10.2341/11-045-1.

18. Cavalli V, Shinohara MS, Ambrose W, Malafaia FM, Pereira PN, Giannini M. Influence of intracoronal bleaching agents on the ultimate strength and ultrastructure morphology of dentine. Int Endod J. 2009 Jul;42(7):568-75. doi: 10.1111/j.1365-2591.2009.01543.x.

19. de Oliveira DP, Teixeira EC, Ferraz CC, Teixeira FB. Effect of intracoronal bleaching agents on dentin microhardness. J Endod. 2007Apr;33(4):460-2. doi: 10.1016/j.joen.2006.08.008.

20. Patel S, Kanagasingam S, Ford TP. External cervical resorption: a review. J Endod. 2009 May;35(5):616-25. doi: 10.1016/j.joen.2009.01.015.

21. Rotstein I, Mor C, Friedman S. Prognosis of intracoronal bleaching with sodium perborate preparations in vitro: 1-year study. J Endod. 1993;19(1):10-2.

22. Weiger R, Kuhn A, Lost C. In vitro comparison of various types of sodium perborate used for intracoronal bleaching of discolored teeth. J Endod. 1994;20(7):338-41. doi: 10.1016/Soo99-2399(06)80096-8.

23. Lou EK, Cathro P, Marino V, Damiani F, Heithersay GS. Evaluation of hydroxyl radical diffusion and acidified thiourea as a scavenger during intracoronal bleaching. J Endod. 2016 Jul;42(7):1126-30. doi: 10.1016/j.joen.2016.04.010. 